\title{
USING DIRECT LINEAR TRANSFORMATION (DLT) METHOD FOR AERIAL PHOTOGRAMMETRY APPLICATIONS
}

\author{
Khalid L. A. EL-ASHMAWY ${ }^{*}$ \\ Department of Civil Engineering, College of Engineering and Islamic Architecture, \\ Umm Al-Qura University, Makkah, Saudi Arabia
}

Received 30 April 2018; accepted 20 September 2018

\begin{abstract}
DLT has gained a wide popularity in close range photogrammetry, computer vision, robotics, and biomechanics. The wide popularity of the DLT is due to the linear formulation of the relationship between image and object space coordinates.

This paper aims to develop a simple mathematical model in the form of self calibration direct linear transformation for aerial photogrammetry applications. Software based on the derived mathematical model has been developed and tested using mathematical photogrammetric data.

The effects of block size, number and location of control points, and random and lens distortion errors on self calibration block adjustments using the derived mathematical model and collinearity equations have been studied. It was found that the accuracy of the results of self calibration block adjustment using the derived mathematical model is, to some extent, comparable to the results with collinearity model.

The developed mathematical model widens the application areas of DLT method to include aerial photogrammetry applications especially when the camera interior and exterior orientations are unknown.
\end{abstract}

Keywords: aerial photogrammetry, DLT, MDLT, self calibration block adjustment, bundle block adjustment.

\section{Introduction}

In topographical photogrammetry, photogrammetrists think most naturally in terms of models produced by pairs of photographs. However, undoubtedly the most flexible approach to block formation and adjustment and to photogrammetry in general is through the use of the bundles of rays produced by individual photographs. In closerange photogrammetry, where multi-station and convergent configurations are possible, the bundle approach can be seen in its most powerful form. The bundle approach is still the most flexible available approach and one that should give the best results.

The adjustment of the bundles in a block of photographs involves the rotation and translation of each bundle in space into such a position that all rays passing through the photographic positions of each control point will intersect at its correct object space position. Furthermore, all rays representing other points, such as a pass point, must intersect at their respective position in the object space.
The method of bundle adjustment makes use of the collinearity condition (Tommaselli \& Junior, 2012; Ghosh, 2005; El-Ashmawy, 1999) or coplanarity condition (ElAshmawy \& Azmi, 2003). The desired parameters (which include object space coordinates of pass and/or tie points, and camera exterior orientation parameters) are adjusted as a result of one simultaneous least squares solution of all the photographs by an iterative method. The iterations are necessary because of the fact that the associated condition equations are non-linear. The results of the bundle adjustment of the block of photographs are camera exterior orientation parameters of each photograph and a listing of the object space coordinates of the measured pass and/or tie points as well as their statistical precision.

The fundamental requirements in bundle adjustment are the estimates of the camera interior and exterior orientations parameters. Furthermore, depending on the specific approach taken, the estimates for object space coordinates of all pass and/or tie points may also be needed. Thus, a bundle procedure should include a feasible method

*Corresponding author. E-mail: khalid85_2002@yahoo.com 
of obtaining the necessary estimated (approximate) values initially. The importance of initial values being close to most probable values of unknowns needs no emphasis. Not only it reduces the number of iterations but ensures fast and accurate results.

Determination of the initial values of camera exterior orientation parameters and the object space coordinates of pass and/or tie points depends mainly on the number of the control points (El-Ashmawy, 1999). Two cases as follows, different in their principles, which have been found in common uses in photogrammetry are:

- CASE A: when the number of the control points is three or more per photograph. This case is a general occurrence in close range photogrammetry. The steps for this method are based on space resection for obtaining the camera exterior orientation parameters for each photo followed by space intersection for obtaining the object space coordinates of new points, points rather than control points. This method depends mainly on using collinearity equations.

- CASE B: when the number of control points is three or more per photogrammetric block. This, generally, occurs in aerial photogrammetry applications. This method is based on an approach similar to analogical procedure for determining the initial values of object space coordinates of pass and/or tie points and using these to determine the initial values of exterior orientation parameters. Coplanarity equation, collinearity equations and seven parameters coordinate transformation are widely used in this method.

The prerequisites for the two above mentioned methods are the prior knowledge of camera interior orientation parameters.

The Direct Linear Transformation (DLT) method was introduced to the photogrammetric community by (Abdel-Aziz \& Karara, 1971). DLT method models the transformation between the comparator or image pixel coordinate system and the object coordinate system as a linear function. DLT can be derived from the standard collinearity equations. The basic equations of DLT are expressed as follows (Abdel-Aziz \& Karara, 2015):

$$
\begin{aligned}
& x=\frac{L_{1} X+L_{2} Y+L_{3} Z+L_{4}}{L_{9} X+L_{10} Y+L_{11} Z+1} ; \\
& y=\frac{L_{5} X+L_{6} Y+L_{7} Z+L_{8}}{L_{9} X+L_{10} Y+L_{11} Z+1},
\end{aligned}
$$

where $x, y$ are the image coordinates, $L_{1}, \ldots, L_{11}$ are the transformation coefficients and $X, Y, Z$ are the object space coordinates of point.

The values of camera interior and exterior orientations are then computed by:

$$
\left[\begin{array}{c}
X_{0} \\
Y_{0} \\
Z_{0}
\end{array}\right]=-\left[\begin{array}{lll}
L_{1} & L_{2} & L_{3} \\
L_{5} & L_{6} & L_{7} \\
L_{9} & L_{10} & L_{11}
\end{array}\right]^{-1}\left[\begin{array}{c}
L_{4} \\
L_{8} \\
1.0
\end{array}\right] ;
$$

$$
\begin{aligned}
& x_{0}=\left(L_{1} L_{9}+L_{2} L_{10}+L_{3} L_{11}\right) /\left(L_{9}^{2}+L_{10}^{2}+L_{11}^{2}\right) ; \\
& y_{0}=\left(L_{5} L_{9}+L_{6} L_{10}+L_{7} L_{11}\right) /\left(L_{9}^{2}+L_{10}^{2}+L_{11}^{2}\right) ; \\
& \omega=\tan ^{-1}\left(-L_{10} / L_{11}\right) ; \\
& \phi=\sin ^{-1}\left(-L_{9} / \sqrt{\left(L_{9}^{2}+L_{10}^{2}+L_{11}^{2}\right)}\right) ; \\
& \kappa=\cos ^{-1}\left(\left(x_{0} L_{9}-L_{1}\right) /\right. \\
& \left.\left(\cos \phi \sqrt{\left(x_{0} L_{9}-L_{1}\right)^{2}+\left(x_{0} L_{10}-L_{2}\right)^{2}+\left(x_{0} L_{11}-L_{3}\right)^{2}}\right)\right) ;
\end{aligned}
$$$$
f=\left(x_{0} L_{9}-L_{1}\right) /\left(\cos \kappa \cdot \cos \phi \sqrt{L_{9}^{2}+L_{10}^{2}+L_{11}^{2}}\right),
$$

where $X_{0}, Y_{0}, Z_{0}, \omega, \phi, \kappa$ are camera exterior orientation parameters, $x_{0}, y_{0}$ image coordinates of the principal point and $f$ is the camera focal length.

DLT has gained a wide popularity in close range photogrammetry, computer vision, robotics, and biomechanics. The wide popularity of the DLT is due to the linear formulation of the relationship between image and object coordinates. Namely the following characteristics are associated with the DLT model: image coordinates can be expressed in a non-orthogonal system with unequal scales, the position of the coordinate system is arbitrary, and the principal distance can be unknown and vary from image to image.

This paper aims to:

- Derive a mathematical formulation based on DLT method for aerial photogrammetry applications;

- Investigate the accuracy of the derived mathematical formulation; and

- Compare between the results of the derived mathematical formulation with the results of collinearity equations for self calibration block adjustment.

\section{The mathematical formulation}

\subsection{The mathematical model for self calibration block adjustment}

The standard DLT equations (Equations $(1,2)$ ) actually contain 10 independent unknown parameters. In other word one of the DLT parameters must be redundant and it is needed to add a non-linear constraint to the system to solve this problem. In this case, the method is called modified direct linear transformation (MDLT) method. In MDLT, one of the parameters is expressed in terms of the other 10 parameters and gives a non-linear constraint which is as follows:

$$
\begin{aligned}
& \left(L_{1} L_{5}+L_{2} L_{6}+L_{3} L_{7}\right)\left(L_{9}^{2}+L_{10}^{2}+L_{11}^{2}\right)= \\
& \left(L_{1} L_{9}+L_{2} L_{10}+L_{3} L_{11}\right)\left(L_{5} L_{9}+L_{6} L_{10}+L_{7} L_{11}\right) .
\end{aligned}
$$

From Equation (4)

$$
\begin{aligned}
L_{1}= & \left(\left(L_{2} L_{10}+L_{3} L_{11}\right)\left(L_{5} L_{9}+L_{6} L_{10}+L_{7} L_{11}\right)-\right. \\
& \left.\left(L_{2} L_{6}+L_{3} L_{7}\right)\left(L_{9}^{2}+L_{10}^{2}+L_{11}^{2}\right)\right) / \\
& \left(L_{5}\left(L_{9}^{2}+L_{10}^{2}+L_{11}^{2}\right)-L_{9}\left(L_{5} L_{9}+L_{6} L_{10}+L_{7} L_{11}\right)\right) .
\end{aligned}
$$


Removing $L_{1}$ from Equations $(1,2)$ leads to:

$$
\begin{aligned}
& x=\left(L_{4} L_{5} L_{10}^{2}+L_{4} L_{5} L_{11}^{2}-X L_{2} L_{6} L_{9}^{2}-X L_{2} L_{6} L_{11}^{2}-\right. \\
& X L_{3} L_{7} L_{9}^{2}-X L_{3} L_{7} L_{10}^{2}+X L_{2} L_{10} L_{5} L_{9}+ \\
& X L_{2} L_{10} L_{7} L_{11}+X L_{3} L_{11} L_{5} L_{9}+X L_{3} L_{11} L_{6} L_{10}+ \\
& Y L_{2} L_{5} L_{10}^{2}+Y L_{2} L_{5} L_{11}^{2}-Y L_{2} L_{9} L_{6} L_{10}- \\
& Y L_{2} L_{9} L_{7} L_{11}+Z L_{3} L_{5} L_{10}^{2}+Z L_{3} L_{5} L_{11}^{2}- \\
& Z L_{3} L_{9} L_{6} L_{10}-Z_{L_{3}} L_{9} L_{7} L_{11}-L_{4} L_{9} L_{6} L_{10}- \\
& \left.L_{4} L_{9} L_{7} L_{11}\right) /(a+b) ; \\
& y=\frac{L_{5} X+L_{6} Y+L_{7} Z+L_{8}}{L_{9} X+L_{10} Y+L_{11} Z+1}
\end{aligned}
$$

where

$$
\begin{aligned}
a= & \left(L_{5} L_{11}^{2}+L_{5} L_{10}^{2}+X L_{9} L_{5} L_{10}^{2}+X L_{9} L_{5} L_{11}^{2}-X L_{9}^{2} L_{6} L_{10}-\right. \\
& \left.X L_{9}^{2} L_{7} L_{11}+Y L_{10}^{3} L_{5}+Y L_{10} L_{5} L_{11}^{2}-Y L_{10}^{2} L_{9} L_{6}\right) ; \\
b= & \left(-Y L_{10} L_{9} L_{7} L_{11}+Z L_{11} L_{5} L_{10}^{2}+Z L_{11}^{3} L_{5}-Z L_{11} L_{9} L_{6} L_{10}-\right. \\
& \left.Z L_{11}^{2} L_{9} L_{7}-L_{9} L_{6} L_{10}-L_{9} L_{7} L_{11}\right) .
\end{aligned}
$$

In Equation (6), the observations are the photo coordinates on an object point. Considering the case of camera calibration, the $x_{p} \& y_{p}$ corrected photo coordinates can be obtained by (Ghosh, 2005):

$$
\left.\begin{array}{l}
x_{p}=x-F(k) \cdot \xi+\Delta x(p) \\
y_{p}=y-F(k) \cdot \eta+\Delta y(p)
\end{array}\right\},
$$

where $x, y$ are the observed photo coordinates;

$$
\begin{aligned}
& \xi=x-x_{0} ; \\
& \eta=y-y_{0} ; \\
& r^{2}=\xi^{2}+\eta^{2} ; \\
& F(k)=L_{12} r^{2}+L_{13} r^{4}+L_{14} r^{6}
\end{aligned}
$$

$=$ function of symmetrical radial lens distortion and $L_{12}, L_{13}, L_{14}$ are its correction coefficients;

$\Delta x(p), \Delta y(p)$ are the decentering lens distortion corrections for $x$ and $y$ photo coordinates, and can be obtained by:

$$
\left.\begin{array}{l}
\Delta x(p)=L_{15}\left(r^{2}+2 \xi^{2}\right)+L_{16} \xi \eta \\
\Delta y(p)=L_{15} \eta \xi+L_{16}\left(r^{2}+2 \eta^{2}\right)
\end{array}\right\},
$$

in which $L_{15}, L_{16}$ are correction coefficients for decentering lens distortion.

Merging Equations $(6,7,8,9)$ leads to:

$$
\begin{aligned}
& x=\left(L_{4} L_{5} L_{10}^{2}+L_{4} L_{5} L_{11}^{2}-X L_{2} L_{6} L_{9}^{2}-X L_{2} L_{6} L_{11}^{2}-X L_{3} L_{7} L_{9}^{2}-\right. \\
& X L_{3} L_{7} L_{10}^{2}+X L_{2} L_{10} L_{5} L_{9}+X L_{2} L_{10} L_{7} L_{11}+X L_{3} L_{11} L_{5} L_{9}+ \\
& X L_{3} L_{11} L_{6} L_{10}+Y L_{2} L_{5} L_{10}^{2}+Y L_{2} L_{5} L_{11}^{2}-Y L_{2} L_{9} L_{6} L_{10}- \\
& Y L_{2} L_{9} L_{7} L_{11}+Z L_{3} L_{5} L_{10}^{2}+Z L_{3} L_{5} L_{11}^{2}-Z L_{3} L_{9} L_{6} L_{10}- \\
& \left.Z_{L_{3}} L_{9} L_{7} L_{11}-L_{4} L_{9} L_{6} L_{10}-L_{4} L_{9} L_{7} L_{11}\right) /(a+b)+ \\
& \xi\left(L_{12} r^{2}+L_{13} r^{4}+L_{14} r^{6}\right)+L_{15}\left(r^{2}+2 \xi^{2}\right)+L_{16} \xi \eta
\end{aligned}
$$

$$
\begin{gathered}
y=\frac{L_{5} X+L_{6} Y+L_{7} Z+L_{8}}{L_{9} X+L_{10} Y+L_{11} Z+1}+\eta\left(L_{12} r^{2}+L_{13} r^{4}+\right. \\
\left.L_{14} r^{6}\right)+L_{15} \eta \xi+L_{16}\left(r^{2}+2 \eta^{2}\right) .
\end{gathered}
$$

The linearized form of Equation (10), for least squares method solution, can be given by:

$$
V+B \cdot \Delta=\varepsilon,
$$

where $\Delta$ is the correction vector to the current values set for the unknowns (the 15 MDLT parameters for each photo and object space coordinates of the new points) in the iterative solution; $B$ is the matrix of the partial derivatives of Equation (10) with respect to the unknowns; $V$ is the residual vector, i.e., the correction vector to the observations; and $\varepsilon$ is the discrepancy vector.

Moreover, additional constraints are suggested to consider supplemental observation equations (El-Ashmawy, 1999; El-Ashmawy \& Azmi, 2003; Ghosh, 2005) arising from a priori knowledge regarding the object space coordinates of the control points in Equation (11). Such supplemental equations can be written as follows:

$$
V^{c}-\Delta^{c}=\varepsilon^{c},
$$

where $\Delta^{c}$ is the vector of observational corrections to the object space coordinates of the control points; and $\varepsilon^{c}$ is the discrepancy vector, between observed values and current (in iterative solution) values of the object space coordinates of the control points.

Observation equations can be obtained by merging Equations (11) and (12) as:

$$
\left.\begin{array}{l}
V+B \cdot \Delta=\varepsilon \\
V^{c}-\Delta^{c}=\varepsilon^{c}
\end{array}\right\},
$$

in which $\Delta$ is the correction vector to the current values set for the unknowns which are the MDLT parameters for each photo and object space coordinates of all points. The other matrices are as defined earlier.

The principle of the least squares method requires the minimizing of the quadratic form $V^{t} \cdot W \cdot V$, where $W$ is the weight matrix whose elements are the weights associated with each of the observations. The least squares solution of an equation similar to Equation (13) can be given as (Mikhail, 1976; Ghosh, 2005):

$$
\Delta=N^{-1} \cdot C,
$$

where

$$
\left.\begin{array}{l}
N=B^{t} \cdot W \cdot B \\
C=B^{t} \cdot W \cdot \varepsilon
\end{array}\right\} .
$$

Variance of unit weight can be computed as:

$$
\hat{\sigma}_{0}^{2}=V^{t} \cdot W \cdot V /(N-U),
$$

where $\hat{\sigma}_{0}^{2}$ is the variance of unit weight; $N$ is the number of observations and evaluated from:

$$
N=3 n_{1}+2 l
$$


in which $n_{1}$ is the number of control points; and $l$ is the number of image points; $U$ is the number of unknown and evaluated from:

$$
U=3 n+15 m,
$$

in which $n$ is the number of object points (control, pass, etc.); and $m$ is the number of photographs.

$$
(N-U)=\text { Degree of freedom. }
$$

\subsection{Computation of the approximate values of $D L T$ parameters and object space coordinates of new points}

For starting the iterative solution of Equation (13) approximate values of unknowns should be known. These unknowns are the object space coordinates of new points and the MDLT parameters for each photo. As has been mentioned earlier, the importance of initial values being close to most probable values of unknowns reduces the number of iterations and ensures fast and accurate results. The suggested method for estimating the values of unknowns is illustrated in Figure 1.

The estimation method of the values of object space coordinates of new points is outlined earlier and explained in (El-Ashmawy, 1999).

Finding the approximate MDTL parameters is an iterative approach. In the first iteration, 16 DTL parameters are solved using the conventional DLT method. Eight control, or known object space coordinates, points are required for each photograph. This is not restricted condition after computing the object space coordinates of all points, as explained in the previous step. From the second iteration, $L_{1}$ can be removed by using the value obtained from the previous iteration and the system is reduced to 15 parameters. The system is solved for 15 parameters. The parameter removed earlier $\left(L_{1}\right)$ is computed based on the

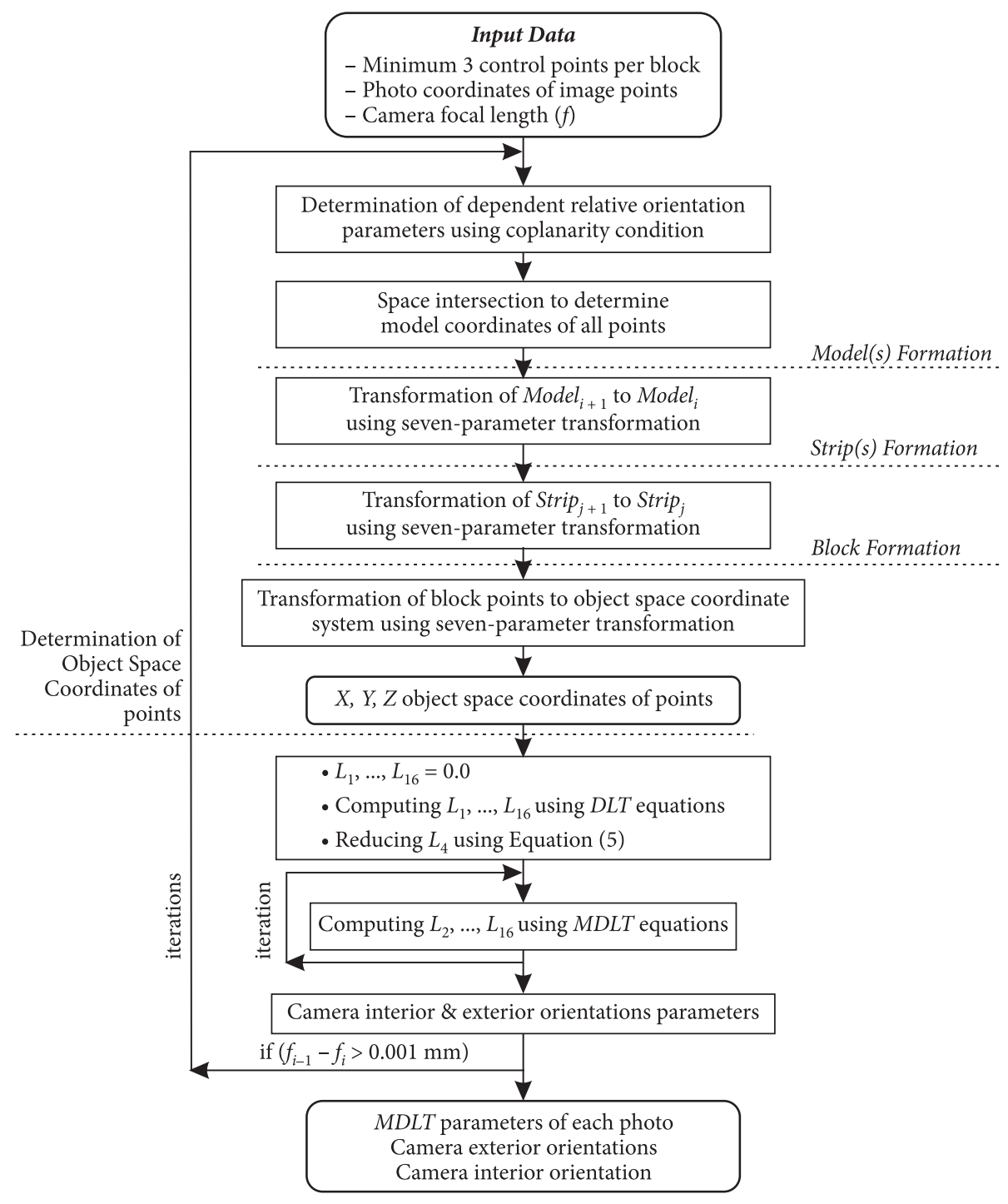

Figure 1. Computation of the initial values of object space coordinates of points, MDLT parameters and camera interior and exterior orientations 
10 estimated parameters using Equation (5). At this stage, the camera exterior and interior orientations parameters can be computed. The second step is repeated till a stable (converged) set of solution is obtained.

Computing the camera focal length is also an iterative solution. After computing the DLT parameters, the camera focal length is determined using Equation (3). The new value of camera focal length is used to determine a new set of object space coordinates of new points, DLT parameters and camera focal length. The solution is repeated till the difference in the values of camera focal length can be neglected.

\section{Developing the necessary software}

The current research includes the development of software for aerotriangulation application utilizing DLT method named as AerDLT software.

The software provides an access to major computational phases of analytical block triangulation. The main functions of the developed software are:

1. Data preparation: It performs the necessary tasks for preparing the data to start block adjustment such as: - Testing the geometry of the input data: It includes testing the availability of each point in at least two photographs and giving complete information about the block adjustment parameters such as number of observations and unknowns, degree of freedom, etc.

- Computation of the initial values of unknowns: It includes the computation of the initial values of DLT parameters and/or object space coordinates of points which are essential for starting the iterative solution. Moreover, the software is able to compute the camera interior and exterior orientations parameters.

2. Iterative least squares method solution for performing the specified task. This includes the computations of the adjusted values of unknowns, residuals of photo and object space coordinates of control points and variance of unit weight.

3. Computation of statistical data: It includes the computation of the necessary data for statistical analysis and error detection (El-Ashmawy, 1999) such as variance of unit weight, adjusted photo coordinates, residuals of photo coordinates, etc.

For automatic processing and representation of the data and results, the software utilises efficient techniques of Data Structuring, Random File Access and Dynamic Memory Allocations (Malik, 2010). The software has been designed to make use of efficient user interfaces (window-driven) for facilitating its execution to the user (Gregory, 1998).

\section{Testing the developed software}

Testing a photogrammetric system is a complex task. It involves, for example, availability of suitable data for testing, decision regarding number and type of tests to be carried out, photogrammetric tasks for which test may be carried out, and many other considerations.

After the completion of the development stages, the software was subjected to a series of tests. These tests presented an opportunity to verify that the developed software satisfies general performance requirements, especially with regard to efficiency, flexibility and feasibility of processing the photogrammetric data.

Mathematical photogrammetric data can be advantageously used for testing of photogrammetric methodologies and systems since in this case error free input data and end results are both known. Testing the developed software, therefore, was carried out by using the mathematically generated blocks of photographs of MATHP software (El-Ashmawy, 1999).

Out of the various mathematical photogrammetric blocks generated, the block having following specifications was used for testing AerDLT:

a. Photograph scale: $1: 1$.

b. Camera format: $230.0 \mathrm{~mm} \times 230.0 \mathrm{~mm}$.

c. Camera focal length: $150.00 \mathrm{~mm}$.

d. Longitudinal and Lateral overlaps: $65 \%$ and $30 \%$ respectively.

e. Total number of points available per model: 18 .

f. Terrain configuration: hilly type with height variation of $25 \%$ of the flying height.

Five different block sizes were generated. The size of the blocks ranged from one model to a size of 5 strips each of 5 photographs.

Since in each photograph, several control points were available, a suitable number with different well distribution pattern were used for testing the methodology of the developed software. In particular the following control point distributions have been used for various determinations:

Pattern I: 4 control points/block.

Pattern II: 9 control points/block.

Pattern III: 6 control points/strip.

Pattern IV: 6 control points/photograph.

For comparing the results of the derived mathematical model, Col_Cal_Consts software (El-Ashmawy \& Azmi, 2003) was used. The mathematical foundation for developing Col_Cal_Consts is based on the collinearity equations with adding constraints to the control points coordinates.

The objectives set for the testing phase were:

- Testing the system error; and

- Testing the feasibility of camera focal length determination method.

\subsection{Testing the system error}

Self calibration block adjustments involve extensive computations and the various steps of which are subjected to computational system error. System error consists of two parts (El-Ashmawy, 1999). The first part of this error is due to rounding off of values during intermediate computations. This part of error may be minimised by using double precision computations as far as possible. The 
second part of the system error occurs due to truncation of higher order terms while forming the linearized observation equations from the non linear condition equations.

To reduce the effect of the number and location of the control points during the testing phase of the system error, Pattern IV was used. The block size was 5 strips each of five photographs. In this case, the block contained 66 and 132 control and check points respectively.

In order to ascertain the accuracy of the results, the root mean square error (RMSE) was computed using the well known formulation:

$$
R M S E=\sqrt{\sum_{i=1}^{n}(\text { known value }- \text { computed value })_{i}^{2} / n} .
$$

The RMSE values for ground coordinates of check points have been obtained. The results showed that the maximum RMSE value is $0.0001 \mu \mathrm{m}$, at photo scale $1: 1$, which is negligible. From above, it is seen that the developed software is free from system error and that it is functional.

\subsection{Testing the feasibility of camera focal length determination method}

Camera focal length is only required for the determination of approximate object space coordinates of new points. In fact the derived mathematical model (Equation (10)) does not need any knowledge of camera interior or exterior orientation parameters.

Elimination of the need of accurate value of camera focal length is the aim of this test. Different tests were carried out by using different values of camera focal length and recording the computed focal length by the software (Figure 1). The tests showed that the software is able to compute the accurate value of the focal length $(150 \mathrm{~mm})$ when the starting value of focal length is within $150 \pm$ $15 \mathrm{~mm}$. This means that the developed methodology is powerful for computing the accurate value of camera focal length from a rough value.

\section{Applications of the developed software for self calibration block adjustment}

The objectives of these applications were:

- Testing the geometric error;

- Studying the effect of the random errors on block adjustment;

- Studying the effect of the lens distortion errors on block adjustment; and

- Studying the effect of random and lens distortion errors on block adjustment.

\subsection{Testing the geometric error}

This error is a result of the effect of the block size, the number and location of the control points and the geometric solution which is provided by the mathematical model itself. The five control points patterns were used for this purpose. The results of the block adjustment for this test are shown in Table 1.

From Table 1 it can be concluded that:

- DLT method is suitable for self calibration block adjustment for a block of photographs of any size.

- The geometric error decreases with the increasing of the number of control points.

- Generally, using 6 control points/strip or more is necessary for overcoming the effects of the geometric error.

\subsection{Studying the effect of the random errors on block adjustment}

The effect of the random errors was tested by numerical simulation as following:

- Generating error free photogrammetric data of blocks of different sizes using MATHP software as explained earlier.

- Generating normally distributed error(s) with arbitrary mean(s) and standard deviation(s) as presented in (El-Ashmawy \& Azmi, 2003). The obtained errors were then applied to the error free photo coordinates and ground coordinates of control points of the generated blocks. The configurations of the used blocks are shown in Table 2.

- To reduce the effect of the geometric error, control points Pattern IV was used in this test.

- Finally, self calibration block adjustments using the derived mathematical model for DLT method and collinearity equations were performed to adjust the available blocks and the results, in the form of standard deviation of unit weight $\left(\hat{\sigma}_{0}\right)$ and $R M S E$ values at control and check points, were obtained and tabulated in Table 3.

From Table 3, the following conclusions can be obtained:

- There is no significant difference between the posterior standard deviation $\left(\hat{\sigma}_{0}\right)$ and the a priori standard deviation $\left(\sigma_{0}\right)$ and hence that the correct simulation assumptions and block adjustment have been achieved.

- The results of DLT method for control points are much better than the results of the collinearity equations especially for the $Z$ coordinates determination.

- Furthermore, there is slight improvement for the accuracy of check points coordinates determination using collinearity equations rather than DLT method.

\subsection{Studying the effect of the lens distortion errors on block adjustment}

As has been mentioned, lens distortion consists of two components: symmetric lens distortion and asymmetric lens distortion. The adopted mathematical model for symmetric lens distortion is given by Equation (8). The asymmetrical lens distortion is due to the lenses decentering 
Table 1. Results of testing the geometric error

\begin{tabular}{|c|c|c|c|c|c|c|c|c|c|c|c|}
\hline \multirow{3}{*}{$\begin{array}{l}\text { Block } \\
\text { Size }\end{array}$} & \multirow{3}{*}{$\begin{array}{l}\text { Control } \\
\text { Points } \\
\text { Pattern }\end{array}$} & \multirow{3}{*}{$\begin{array}{l}\text { Control } \\
\text { Points }\end{array}$} & \multirow{3}{*}{$\begin{array}{l}\text { Check } \\
\text { Points }\end{array}$} & \multirow{3}{*}{$\begin{array}{l}\text { Image } \\
\text { Points }\end{array}$} & \multirow{3}{*}{$\begin{array}{l}\text { Degree } \\
\text { of } \\
\text { Freedom }\end{array}$} & \multicolumn{6}{|c|}{ RMSE Values $(\mu \mathrm{m})^{*}$} \\
\hline & & & & & & \multicolumn{3}{|c|}{ Control Points } & \multicolumn{3}{|c|}{ Check Points } \\
\hline & & & & & & $\mathrm{X}$ & $\mathrm{Y}$ & $\mathrm{Z}$ & $\mathrm{X}$ & $\mathrm{Y}$ & $\mathrm{Z}$ \\
\hline \multirow{2}{*}{ Model } & I & 4 & 14 & 36 & 0 & 0.0 & 0.0 & 0.0 & 3.0 & 3.0 & 5.0 \\
\hline & III & 6 & 12 & 36 & 6 & 0.0 & 0.0 & 0.0 & 0.0 & 0.0 & 0.0 \\
\hline \multirow{4}{*}{1 Strip } & I & 4 & 41 & 117 & 36 & 0.0 & 0.0 & 0.0 & 7.0 & 2.0 & 11.0 \\
\hline & II & 9 & 36 & 117 & 51 & 0.0 & 0.0 & 0.0 & 0.0 & 0.0 & 0.0 \\
\hline & III & 6 & 39 & 117 & 42 & 0.0 & 0.0 & 0.0 & 0.0 & 0.0 & 0.0 \\
\hline & IV & 15 & 30 & 117 & 69 & 0.0 & 0.0 & 0.0 & 0.0 & 0.0 & 0.0 \\
\hline \multirow{4}{*}{2 Strip } & I & 4 & 77 & 246 & 111 & 0.0 & 0.0 & 0.0 & 1.0 & 1.0 & 2.0 \\
\hline & II & 9 & 72 & 246 & 126 & 0.0 & 0.0 & 0.0 & 0.0 & 0.0 & 0.0 \\
\hline & III & 9 & 72 & 246 & 126 & 0.0 & 0.0 & 0.0 & 0.0 & 0.0 & 0.0 \\
\hline & IV & 27 & 54 & 246 & 180 & 0.0 & 0.0 & 0.0 & 0.0 & 0.0 & 0.0 \\
\hline \multirow{4}{*}{3 Strip } & I & 4 & 113 & 375 & 186 & 0.0 & 0.0 & 0.0 & 1.0 & 3.0 & 3.0 \\
\hline & II & 9 & 108 & 375 & 201 & 0.0 & 0.0 & 0.0 & 1.0 & 1.0 & 1.0 \\
\hline & III & 12 & 105 & 375 & 210 & 0.0 & 0.0 & 0.0 & 0.0 & 0.0 & 0.0 \\
\hline & IV & 39 & 78 & 375 & 291 & 0.0 & 0.0 & 0.0 & 0.0 & 0.0 & 0.0 \\
\hline \multirow{4}{*}{4 Strip } & I & 4 & 149 & 504 & 261 & 0.0 & 0.0 & 0.0 & 2.0 & 2.0 & 7.0 \\
\hline & II & 9 & 144 & 504 & 276 & 0.0 & 0.0 & 0.0 & 1.0 & 1.0 & 4.0 \\
\hline & III & 15 & 138 & 504 & 294 & 0.0 & 0.0 & 0.0 & 0.0 & 0.0 & 0.0 \\
\hline & IV & 51 & 102 & 504 & 402 & 0.0 & 0.0 & 0.0 & 0.0 & 0.0 & 0.0 \\
\hline \multirow{4}{*}{5 Strip } & I & 4 & 185 & 633 & 336 & 0.0 & 0.0 & 0.0 & 2.0 & 2.0 & 4.0 \\
\hline & II & 9 & 180 & 633 & 351 & 0.0 & 0.0 & 0.0 & 1.0 & 5.0 & 3.0 \\
\hline & III & 18 & 171 & 633 & 378 & 0.0 & 0.0 & 0.0 & 0.0 & 0.0 & 0.0 \\
\hline & IV & 63 & 126 & 633 & 513 & 0.0 & 0.0 & 0.0 & 0.0 & 0.0 & 0.0 \\
\hline
\end{tabular}

${ }^{*}$ Values at Photo Scale 1:1

Table 2. Configurations for mathematical photogrammetric blocks of photographs

\begin{tabular}{|c|c|c|c|c|c|c|c|c|c|c|c|c|c|c|c|c|c|c|}
\hline \multirow{4}{*}{$\begin{array}{l}\text { Block } \\
\text { Title }\end{array}$} & \multicolumn{3}{|c|}{ Block Size } & \multicolumn{2}{|c|}{$\begin{array}{c}\text { Ground } \\
\text { Points }\end{array}$} & \multirow{4}{*}{ 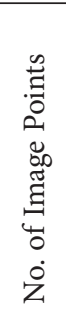 } & \multicolumn{12}{|c|}{ Random Errors $(\mu \mathrm{m})^{*}$} \\
\hline & \multirow{3}{*}{ 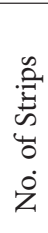 } & \multirow{3}{*}{ 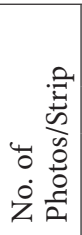 } & \multirow{3}{*}{ 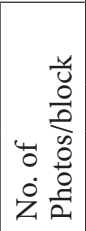 } & \multirow{3}{*}{$\begin{array}{l}0 \\
.0 \\
0 \\
0 \\
0 \\
0 \\
0 \\
0 \\
0\end{array}$} & \multirow{3}{*}{ 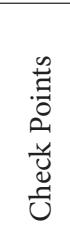 } & & \multicolumn{3}{|c|}{$\begin{array}{c}\text { Photo } \\
\text { Coordinates }\end{array}$} & \multicolumn{9}{|c|}{ Ground Coordinates of Control Points } \\
\hline & & & & & & & \multirow[b]{2}{*}{ జ్ } & \multirow[b]{2}{*}{ 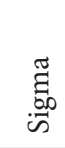 } & \multirow[b]{2}{*}{$\sum_{\Sigma}^{\Xi}$} & \multicolumn{3}{|c|}{$\mathrm{X}$} & \multicolumn{3}{|c|}{$\mathrm{Y}$} & \multicolumn{3}{|c|}{$\mathrm{Z}$} \\
\hline & & & & & & & & & & $\begin{array}{l}\mathscr{\infty} \\
\tilde{\Xi} \\
\approx\end{array}$ & $\begin{array}{l}\tilde{\Xi} \\
\tilde{\omega} \\
\tilde{\omega}\end{array}$ & $\sum_{\Sigma}^{\Xi}$ & $\begin{array}{l}\mathscr{\infty} \\
\approx \\
\approx\end{array}$ & $\underset{\tilde{\Xi}}{\tilde{\omega}}$ & 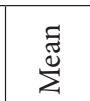 & 芯 & ש్ & $\sum_{\Sigma}^{\Xi}$ \\
\hline Model & 1 & 2 & 2 & 6 & 12 & 36 & $+/-8$ & 3.48 & 0.00 & $+/-5$ & 3.56 & 0.00 & $+/-3$ & 2.16 & 0.00 & $+/-8$ & 4.80 & 0.00 \\
\hline 1 Strip & 1 & 5 & 5 & 15 & 30 & 117 & $+/-10$ & 3.70 & 0.0 & $+/-6$ & 3.70 & -0.07 & $+/-7$ & 3.67 & -0.13 & $+/-10$ & 6.581 & -0.13 \\
\hline 2 Strips & 2 & 5 & 10 & 27 & 54 & 246 & $+/-7$ & 2.16 & 0.0 & $+/-3$ & 1.86 & 0.04 & $+/-6$ & 3.41 & -0.22 & $+1-6$ & 3.37 & 0.15 \\
\hline 3 Strips & 3 & 5 & 15 & 39 & 78 & 375 & $+/-9$ & 3.20 & 0.0 & $+/-5$ & 2.54 & -0.05 & $+/-5$ & 2.78 & -0.05 & $+1-6$ & 3.97 & -0.03 \\
\hline 4 Strips & 4 & 5 & 20 & 51 & 102 & 504 & $+/-9$ & 3.28 & 0.0 & $+/-5$ & 2.19 & -0.06 & $+/-7$ & 3.15 & -0.02 & $+/-8$ & 4.12 & -0.08 \\
\hline 5 Strips & 5 & 5 & 25 & 63 & 126 & 633 & $+/-9$ & 3.37 & 0.0 & $+/-6$ & 1.89 & -0.06 & $+/-8$ & 3.50 & -0.03 & $+1-9$ & 4.53 & 0.00 \\
\hline
\end{tabular}

* Values at Photo Scale 1:1 
and its modeling is given by Equation (9). The lens distortion errors were introduced to the blocks of mathematical photographs as follows:

- Generating error free photogrammetric data of blocks of different sizes using MATHP software.

- Assigning values for the lens distortion coefficients and generating errors in the range of $50 \mu \mathrm{m}$ using Equations (8) and (9).

- Adding the generated errors to the error free photo coordinates

- Reducing the effect of the geometric error by using control points Pattern IV.

The results of self calibration block adjustments are shown in Table 4.

The following observations can be drawn from Table 4:

DLT method is suitable for compensating the lens distortion error for a block of photographs of any size.

The results of the collinearity equations are slightly better than the results of DLT method especially for the determination of $\mathrm{Z}$ coordinates of check points.

\subsection{Studying the effect of random and lens distortion errors on block adjustment}

In this case, error free photogrammetric data of blocks of different sizes using MATHP software were generated and random and lens distortion errors were generated and applied to the error free photo coordinates and ground coordinates of control points of the generated blocks as explained earlier.

Table 5 illustrates the results of self calibration block adjustments for this case.

From Tables 3 and 5 the following conclusions can be drawn:

The results in both tables are identical. This means that the lens distortion errors are totally compensated.

Table 3. Results of self calibration block adjustment (Case of random errors only)

\begin{tabular}{|c|c|c|c|c|c|c|c|c|c|c|}
\hline \multirow{3}{*}{ Method } & \multirow{3}{*}{$\begin{array}{l}\text { Block } \\
\text { Title }\end{array}$} & \multirow{3}{*}{$\begin{array}{l}\text { Degree of } \\
\text { Freedom }\end{array}$} & \multirow{3}{*}{$\sigma_{0}$} & \multirow{3}{*}{$\hat{\sigma}_{0}$} & \multicolumn{6}{|c|}{ RMSE Values for Ground Coordinates $(\mu \mathrm{m})^{*}$} \\
\hline & & & & & \multicolumn{3}{|c|}{ Control Points } & \multicolumn{3}{|c|}{ Check Points } \\
\hline & & & & & $X$ & $Y$ & $Z$ & $X$ & $Y$ & $Z$ \\
\hline \multirow{6}{*}{ DLT } & Model & 006 & 1.00 & 0.92 & 0.30 & 0.20 & 0.20 & 8.50 & 11.6 & 19.70 \\
\hline & 1 Strip & 069 & 1.00 & 1.11 & 1.90 & 1.90 & 3.00 & 6.40 & 5.80 & 13.00 \\
\hline & 2 Strip & 168 & 1.00 & 1.00 & 0.80 & 2.10 & 0.80 & 3.20 & 3.70 & 7.70 \\
\hline & 3 Strip & 267 & 1.00 & 0.98 & 1.10 & 1.70 & 1.40 & 3.10 & 4.10 & 8.80 \\
\hline & 4 Strip & 366 & 1.00 & 0.98 & 0.90 & 2.10 & 1.40 & 3.40 & 4.40 & 9.30 \\
\hline & 5 Strip & 465 & 1.00 & 0.97 & 0.70 & 1.90 & 1.90 & 3.60 & 4.00 & 9.30 \\
\hline \multirow{6}{*}{$\begin{array}{l}\text { Colli- } \\
\text { nearity } \\
\text { Equation }\end{array}$} & Model & 019 & 1.00 & 1.12 & 2.78 & 2.27 & 4.16 & 7.67 & 5.52 & 11.36 \\
\hline & 1 Strip & 109 & 1.00 & 1.11 & 2.54 & 2.74 & 5.26 & 3.05 & 3.34 & 6.51 \\
\hline & 2 Strip & 265 & 1.00 & 1.03 & 1.30 & 2.00 & 2.84 & 1.90 & 2.20 & 5.20 \\
\hline & 3 Strip & 421 & 1.00 & 1.02 & 2.00 & 1.90 & 3.03 & 3.10 & 2.95 & 6.79 \\
\hline & 4 Strip & 577 & 1.00 & 1.01 & 1.90 & 1.80 & 3.50 & 2.95 & 3.16 & 7.84 \\
\hline & 5 Strip & 733 & 1.00 & 1.08 & 1.80 & 2.16 & 3.21 & 3.19 & 3.50 & 8.15 \\
\hline
\end{tabular}

* Values at Photo Scale 1:1.

Table 4. Results of self calibration block adjustment (Case of lens distortions errors only)

\begin{tabular}{|c|c|c|c|c|c|c|c|c|}
\hline \multirow{3}{*}{ Method } & \multirow{3}{*}{ Block Title } & \multirow{3}{*}{$\begin{array}{l}\text { Degree of } \\
\text { Freedom }\end{array}$} & \multicolumn{6}{|c|}{$R M S E$ Values for Ground Coordinates $(\mu \mathrm{m})^{*}$} \\
\hline & & & \multicolumn{3}{|c|}{ Control Points } & \multicolumn{3}{|c|}{ Check Points } \\
\hline & & & $\mathrm{X}$ & $\mathrm{Y}$ & $\mathrm{Z}$ & $\mathrm{X}$ & $\mathrm{Y}$ & $\mathrm{Z}$ \\
\hline DLT & $\begin{array}{l}\text { Model } \\
1 \text { Strip } \\
2 \text { Strip } \\
3 \text { Strip } \\
4 \text { Strip } \\
5 \text { Strip }\end{array}$ & $\begin{array}{l}006 \\
069 \\
168 \\
267 \\
366 \\
465\end{array}$ & $\begin{array}{l}0.00 \\
0.00 \\
0.00 \\
0.00 \\
0.00 \\
0.00\end{array}$ & $\begin{array}{l}0.00 \\
0.00 \\
0.00 \\
0.00 \\
0.00 \\
0.00\end{array}$ & $\begin{array}{l}0.00 \\
0.00 \\
0.00 \\
0.00 \\
0.00 \\
0.00\end{array}$ & $\begin{array}{l}0.10 \\
0.00 \\
0.00 \\
0.10 \\
0.00 \\
0.00\end{array}$ & $\begin{array}{l}0.20 \\
0.10 \\
0.00 \\
0.00 \\
0.00 \\
0.00\end{array}$ & $\begin{array}{l}0.30 \\
0.10 \\
0.10 \\
0.10 \\
0.10 \\
0.10\end{array}$ \\
\hline $\begin{array}{l}\text { Collinearity } \\
\text { Equation }\end{array}$ & $\begin{array}{l}\text { Model } \\
1 \text { Strip } \\
2 \text { Strip } \\
3 \text { Strip } \\
4 \text { Strip } \\
5 \text { Strip }\end{array}$ & $\begin{array}{l}019 \\
109 \\
265 \\
421 \\
577 \\
733\end{array}$ & $\begin{array}{l}0.00 \\
0.00 \\
0.00 \\
0.00 \\
0.00 \\
0.00\end{array}$ & $\begin{array}{l}0.00 \\
0.00 \\
0.00 \\
0.00 \\
0.00 \\
0.00\end{array}$ & $\begin{array}{l}0.00 \\
0.00 \\
0.00 \\
0.00 \\
0.00 \\
0.00\end{array}$ & $\begin{array}{l}0.10 \\
0.00 \\
0.00 \\
0.00 \\
0.00 \\
0.00\end{array}$ & $\begin{array}{l}0.10 \\
0.00 \\
0.00 \\
0.00 \\
0.00 \\
0.00\end{array}$ & $\begin{array}{l}0.30 \\
0.00 \\
0.00 \\
0.00 \\
0.00 \\
0.00\end{array}$ \\
\hline
\end{tabular}

\footnotetext{
* Values at Photo Scale 1:1.
} 
Table 5. Results of self calibration block adjustment (Case of random and lens distortions errors)

\begin{tabular}{|c|c|c|c|c|c|c|c|c|}
\hline \multirow{3}{*}{ Method } & \multirow{3}{*}{ Block Title } & \multirow{3}{*}{$\begin{array}{l}\text { Degree of } \\
\text { Freedom }\end{array}$} & \multicolumn{6}{|c|}{ RMSE Values for Ground Coordinates $(\mu \mathrm{m})^{*}$} \\
\hline & & & \multicolumn{3}{|c|}{ Control Points } & \multicolumn{3}{|c|}{ Check Points } \\
\hline & & & $\mathrm{X}$ & $\mathrm{Y}$ & $\mathrm{Z}$ & $\mathrm{X}$ & $\mathrm{Y}$ & $\mathrm{Z}$ \\
\hline DLT & $\begin{array}{l}\text { Model } \\
1 \text { Strip } \\
2 \text { Strip } \\
3 \text { Strip } \\
4 \text { Strip } \\
5 \text { Strip }\end{array}$ & $\begin{array}{l}006 \\
069 \\
168 \\
267 \\
366 \\
465\end{array}$ & $\begin{array}{l}0.30 \\
1.90 \\
0.80 \\
1.10 \\
0.90 \\
0.70\end{array}$ & $\begin{array}{l}0.20 \\
1.90 \\
2.10 \\
1.70 \\
2.10 \\
1.90\end{array}$ & $\begin{array}{l}0.20 \\
3.00 \\
0.80 \\
1.40 \\
1.40 \\
1.90\end{array}$ & $\begin{array}{l}8.50 \\
6.40 \\
3.20 \\
3.10 \\
3.40 \\
3.60\end{array}$ & $\begin{array}{l}11.6 \\
5.80 \\
3.70 \\
4.10 \\
4.40 \\
4.00\end{array}$ & $\begin{array}{c}19.70 \\
13.00 \\
7.70 \\
8.80 \\
9.30 \\
9.30\end{array}$ \\
\hline $\begin{array}{l}\text { Collinearity } \\
\text { Equation }\end{array}$ & $\begin{array}{l}\text { Model } \\
1 \text { Strip } \\
2 \text { Strip } \\
3 \text { Strip } \\
4 \text { Strip } \\
5 \text { Strip }\end{array}$ & $\begin{array}{l}019 \\
109 \\
265 \\
421 \\
577 \\
733\end{array}$ & $\begin{array}{l}2.78 \\
2.54 \\
1.30 \\
2.00 \\
1.90 \\
1.80\end{array}$ & $\begin{array}{l}2.27 \\
2.74 \\
2.00 \\
1.90 \\
1.84 \\
2.17\end{array}$ & $\begin{array}{l}4.16 \\
5.26 \\
2.80 \\
3.03 \\
3.50 \\
3.21\end{array}$ & $\begin{array}{l}7.67 \\
3.05 \\
1.90 \\
3.10 \\
2.95 \\
3.19\end{array}$ & $\begin{array}{l}5.52 \\
3.34 \\
2.20 \\
2.95 \\
3.16 \\
3.50\end{array}$ & $\begin{array}{c}11.36 \\
6.51 \\
5.20 \\
6.79 \\
7.84 \\
8.15\end{array}$ \\
\hline
\end{tabular}

* Values at Photo Scale 1:1.

The derived mathematical models are suitable for self calibration block adjustment for a block of photographs of any size.

The results of self calibration block adjustment using the collinearity equations with constraints are slightly better than the results for using DLT method.

\section{Conclusions}

In this paper a simple mathematical model in the form of self calibration direct linear transformation for aerial photogrammetry applications has been derived. Unlike the conventional collinearity model, it does not need the known interior orientation nor the exterior orientation parameters. Therefore the derived mathematical model can be used when the necessary information for the collinearity model is not available.

The test results show that the aerotriangulation results with the derived mathematical model is, to some extent, comparable to the results with collinearity model.

The developed software is able to provide the conventional aerotriangulation procedures with accurate/approximate values of camera interior and exterior orientations parameters, and object space coordinates of points.

The developed software is a general purpose photogrammetric software which can be used for both aerial and close range photogrammetry applications.

This paper shows the necessity for the mathematical photogrammetric data for testing the photogrammetric methods and softwares.

\section{References}

Abdel-Aziz, Y. I., \& Karara, H. M. (1971). Direct linear transformation into object space coordinates in close-range photogrammetry. In Proceedings Symposium on Close Range Photogrammetry (pp. 1-18). Urbana, Illinois.

Abdel-Aziz, Y. I., \& Karara, H. M. (2015). Direct linear transformation from comparator coordinates into object space coordinates in close-range. Photogrammetry. Photogrammetric Engineering \& Remote Sensing, 81(2), 103-107. https://doi.org/10.14358/PERS.81.2.103

El-Ashmawy, K. (1999). A cost-effective photogrammetric system for engineering applications (Doctoral dissertation). Department of Civil Engineering, University of Roorkee, Roorkee, India.

El-Ashmawy, K., \& Azmi, M. (2003). Photogrammetric simultaneous and self calibration block adjustments using coplanarity condition. Engineering Research Journal, 87, 85-101.

Ghosh, S. K. (2005). Fundamentals of computational photogrammetry. New Delhi, India: Concept Publishing Company.

Gregory, K. (1998). Special edition using visual C++ 6. Que, USA.

Malik, D. S. (2010). Data structures using C++ (2 nd ed.). USA: Cengage Learing, Inc.

Mikhail, E. M. (1976). Observations and least squares. New York, U.S.A.: IEP-A Dun-Donnelly publishers.

Tommaselli, A. M. G., \& Junior, J. M. (2012). Bundle block adjustment of CBERS-2B HRC imagery combining control points and lines. Photogrammetrie - Fernerkundung - Geoinformation, 2, 129-139(11). https://doi.org/10.1127/1432-8364/2012/0107 